\title{
BrightSide: Creative Learning Approach
}

\author{
Jyotsna Dusane \\ Student \\ Department of Computer \\ Engineering, VESIT
}

\author{
Sonal Misal \\ Student \\ Department of Computer \\ Engineering, VESIT
}

\author{
Priyanka Patil \\ Student \\ Department of Computer \\ Engineering, VESIT
}

\author{
Vidya Katara \\ Student \\ Department of Computer \\ Engineering, VESIT
}

\author{
Sunita Suralkar \\ Assistant Professor \\ Department of Computer \\ Engineering, VESIT
}

\begin{abstract}
Nowadays, from Mobile Augmented games to entertainment applications, from online shopping to Industry solutions, Augmented Reality has gotten one of the most utilized advancements. The paper explores the field of augmented reality, where the main focus is on education. This research mainly focuses on 3 to 5 age groups i.e. for kindergarten students. The BrightSide application is designed for kids that enable the students to have a creative learning experience to overcome any barrier between theoretical and practical knowledge. BrightSide includes features like alphabets, numbers, flowers and animals, games, and quizzes, stories in augmented reality that will make them excited.
\end{abstract}

\section{Keywords}

Augmented Reality, Unity3D, Vuforia, Image Target, Marker Based, Education, Illustration

\section{INTRODUCTION}

Traditional methods of education as we all realize it is becoming a thing of the past. They are becoming more and more digital and driven by technological innovation.Augmented reality in education can be used for a number of purposes. It helps the students to acquire, process, and remember the information very easily. Also AR makes learning itself more engaging and fun.

It is not limited to any single age group or any level of education. It can be used well at all learning levels.Augmented reality has the ability to replace paper textbooks, physical models and posters. Provide students with portable and cheaper learning materials.By using this education becomes more easy and interactive.

Augmented Reality is young technology and is the way of future technologies and innovations. It has a large number of applications in the fields of medical, marketing, advertisement, entertainment, education and training, industrial design , manufacturing and maintenance, architecture, navigation, emergency, interactive gaming and tourism.

Scripting is the most powerful aspect of unity $3 \mathrm{D}$ and has the advantage of quick prototyping and better organization of code i.e. we can use it to handle user input, manipulate objects in the scene, detect collisions etc. Unity supports two programming languages: $\mathrm{C \#}$ and Unity script. Everything from the creation of $3 \mathrm{D}$ models to the enhancement of features like Scaling, rotation, Zooming etc. and showing the straightforward movements of 3D models, applying materials require the use of scripts.

BrightSide is an Augmented Reality based application and can be used by students to learn concepts in an augmented manner. This will help students to see the three dimensional representation of the entity they're learning about. This Software will allow students to greatly extend their understanding of the concepts easily and way better than just seeing the $2 \mathrm{~d}$ pictorial format of the subject. This application has 5 categories i.e.ARBook, Education, Quizzes, Games, and Videos. In the ARBook section students will be able to see some pictures like house, car, trees, etc. in augmented form. In the Education categories, some new and interesting features like alphabets, numbers, flowers, animals will be shown in augmented form with the background Audio. Then there also will be fun games and quizzes and Kid's stories, Yoga videos for children. Basic requirement of the software will be an Android phone or tablet on which BrightSide will run and if the user wants to enhance the experience then VR glasses can also be used.

The major motivation for developing this project is to make classes more interactive and allow learners to focus more on practice instead of just theory as AR in education helps through visualization and full immersion in the subject, students can achieve the best results.

The paper is organized in the following manner: Section 2explains literature survey, Section 3- briefly explains the proposed system, Section 4- explains working module of project , Section 5- explains Implementation details the procedure for creating an app based on augmented reality, the hardware and software requirements, section 6- is proposed algorithm, Section 7- based on the results, Section 8 - explains conclusion for the entire application and future work to modify and enhance the applications for education and other related areas.

\section{LITERATURE SURVEY}

They [1] have explained the types of flashcards in detail. They made a research that Using Augmented Reality Flashcards to Learn Vocabulary in Early Childhood Education is the right decision or not. Will this method of study be helpful for students of the age 5 -6 year? Invitation letter was sent to a kindergarten in Macau which follows the Cambridge curriculum. There were 98 children, aged between 5 and 6 years. The classes were divided into two groups-the experimental group and the control group based on the results 
of the pre-test scores.

They conducted learning activities for experimental and control groups. Learning activities consisted of presentation, in exploration the experimental group used AR flashcards and the control group used traditional paper flashcards, after that they conducted the test and compared the results of both the groups. They found there was a significant difference in the learning scores of the students who use AR flashcards instead of traditional flashcards.

This [2] Shows usage of augmented reality which can permit individual experiments based on abstract models which could be useful in the case of personalised learning. Explained how the mobile Augmented Reality (MAR) has become a useful tool for educators and learners nowadays. They gave a brief overview of "educational affordances for mobile augmented reality" with the help of charts.

Visualization skill is the ability to mentally generate and interpret three dimensional images. Due to absence of proper visualization skills, it is often to see a larger gap between the fresher's students in terms of their visualization skills. So mainly the aim of their application is to increase perception and spatial visualization skills among students.

This app [3] provides guidelines about Colouring activity. This application is presented as a new type of interactive colouring book with augmented reality for kids to learn how to use colouring tools and explain in brief how the live pipeline in mobile applications works with the help of diagram. This application has an Issue on image marker detection on coloured pages. They planned to use deep learning to improve the detection of coloured marker.

The goal of this research paper [5] was to comprehend the effect of an AR portable application on the learning inspiration of undergrad wellbeing science understudies at UCT. The literature survey showed that there is deficient research on the effect of utilizing versatile AR in instruction, and there is space to investigate the capability of AR to improve understudy learning inspiration and add to improved scholarly accomplishment. The literature review summed up various concepts which led to developing the research questions that were based on the attention, relevance, confidence, and satisfaction model of motivational design.

This journal [6] says inspiration, happiness and curiosity are significant elements for any sort of education game. Augmented reality innovation and tangible interfaces are very much acknowledged by the present Primary School youngsters and by their teachers also. Cooperation's among teachers and kids with AR framework will improve the inspiration and joint effort. By using appropriate instructional methodologies, AR may furnish students with various advantages also, leading to powerful learning encounters. The AR innovation likewise gives the occasions to make education experience that are additionally engaging and attractive.

\section{PROPOSED SYSTEM}

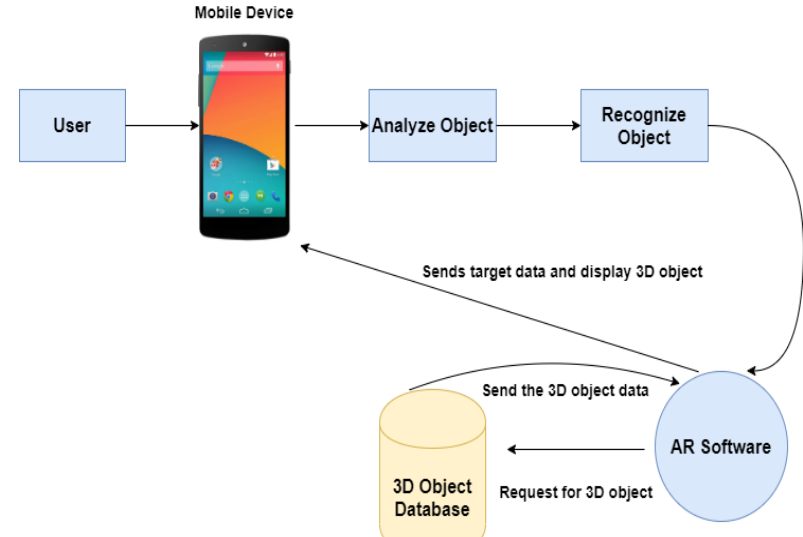

Fig 1: Block Diagram

In this figure firstly feed an image from the camera of a mobile device. Then this image will be analysed and recognized as an object. This module generates binary images i.e. a digital image that has two possible values for every pixel. Typically the 2 colours used for a binary image are black and white. These binary images are provided as an input to the Image Processing Module which are processed using an image processing technique to detect the AR Marker.

Detection of AR Markers is important to work out the position, where to put the virtual object. Once the AR marker is found, its location is provided as input to the tracking module. After that calculated pose is provided to renderingmodule. The renderer uses the calculated pose to combine the real image and the virtual component, and renders the enlarged image on the monitor of the mobile device.

\section{WORKING MODULE}

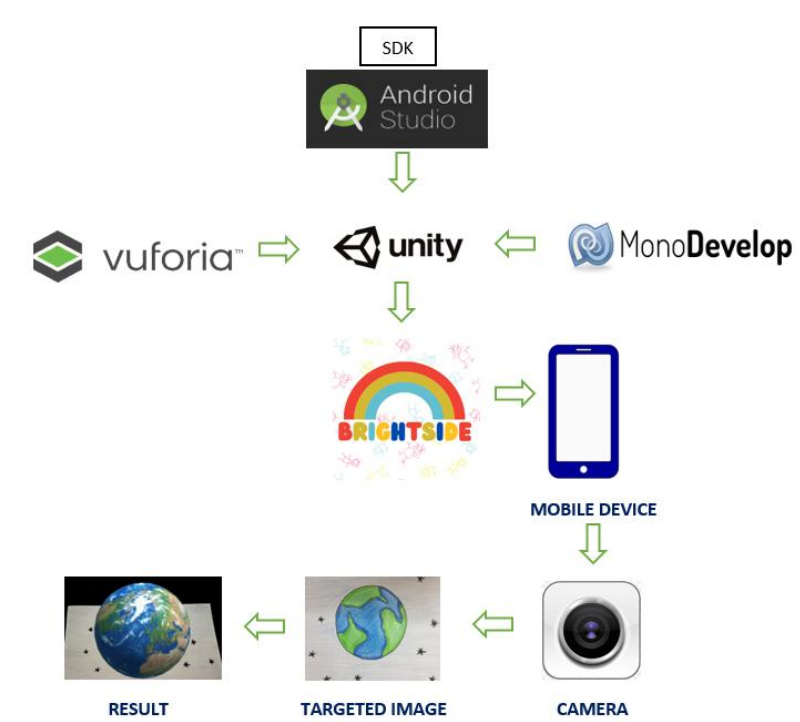

Fig 2: Proposed Model

The proposed model is based on tools such as: Vuforia, Unity and Android Studio along with the interaction of the markers and their output with the object in 3D.

Vuforia created a database that saved the images used as markers, and then imported the Vuforia SDK and Android Studio SDK (so the application has been exported as an Android application package) into the Unity development platform. 
Subsequently, proceed to program the necessary scripts in the IDE, after finishing coding and creating the scenes of the application, proceed to export, create and save the application. Then install it and launch it on the mobile device to make room for the device camera. When the phone camera is pointed at the marker, the device camera will display a threedimensional object.

\section{IMPLEMENTATION DETAILS}

Implementation refers to the creation of applications or execution plans, ideas, models, projects, specifications, standards, algorithms or guidelines.InBrightSide following tools or components are used:

\subsection{Software}

The first stage is the feature detection method, used to detect markers on which augmented form of a particular image will be shown. The second stage restores a $3 \mathrm{D}$ representation of Real image from 3D database and augmented image will be rendered on the display screen of the device.

Software's used for BrightSide are Unity 3D, Vuforia.

\subsubsection{Vuforia Engine}

Vuforia is a mobile augmented reality software development kit that allows you to create augmented reality applications. It uses computer vision technology to detect and track flat images and 3D objects in real time.

\subsubsection{Unity}

It is a cross-platform game engine developed by Unity Technologies and first announced and released at the Apple Inc. Global Developers Conference in June 2005. As the exclusive game engine of Mac OS X.As of 2018, the engine has been expanded to more than 25 platforms. The engine can be used to create 3D, 2D, VR and augmented reality games, as well as assimilation and other experiences.

TABLE 1. Software requirements for BrightSide Application

\section{No. Software Purpose of the software}

1. Unity(2017.2.5f1) Platform to do animation, change scene, scripting and AR camera tool

2. Vuforia SDK(6.6) To handle AR database, image target and license key manager

\subsection{Hardware}

Augmented Reality applications can be implemented by varied technologies such as desktop computers, hand held devices, optical projection systems and so on. BrightSide requires only one hardware device that can either be a smart phone or tablet with pre-installed AR application as well as focuses specifically on the android devices.
TABLE 2. Hardware requirements for BrightSide Application

\section{No. Hardware Purpose of the hardware}

1. Smart phones To test the application

2. Laptop To do the programming

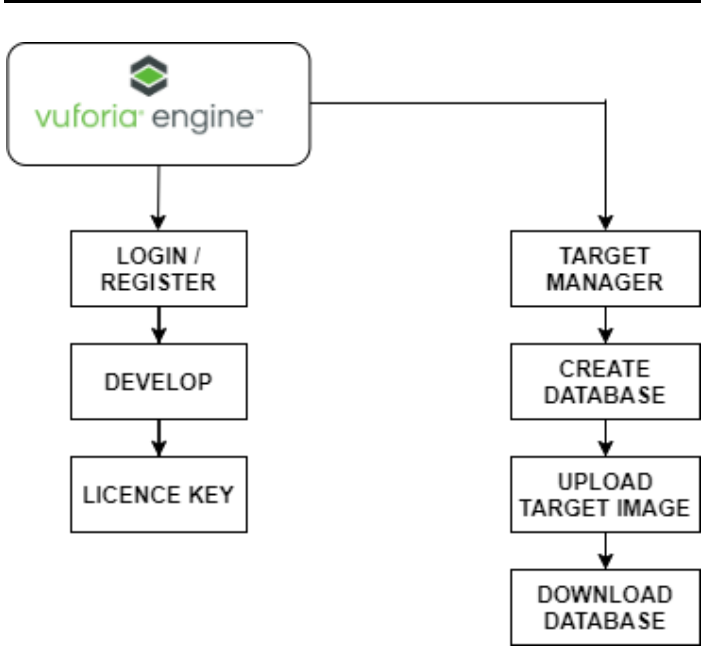

Fig 3: Working of Vuforia

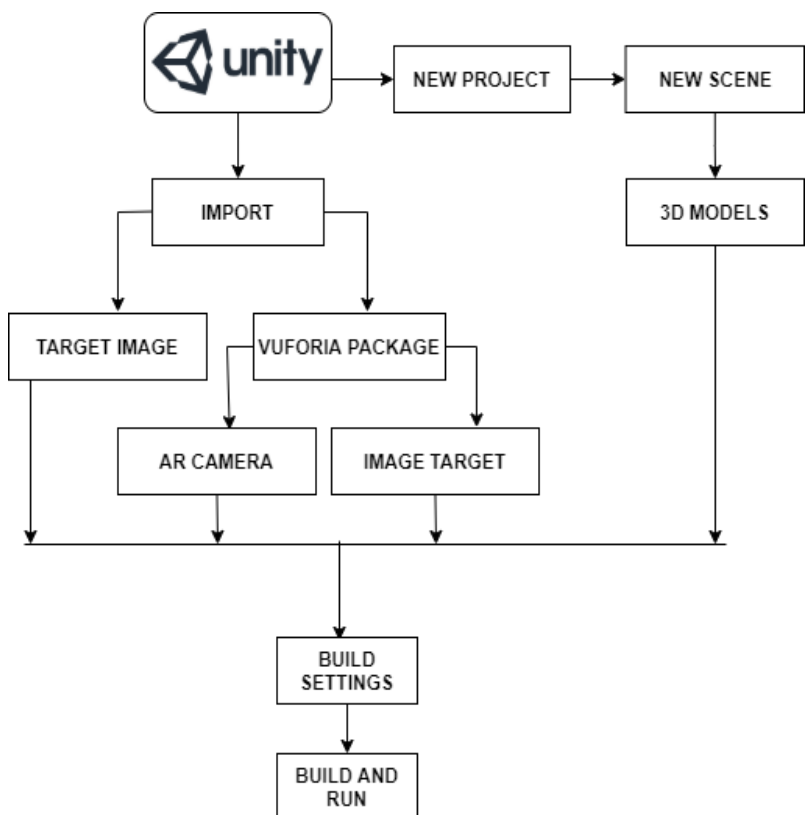

Fig 4: Working of Unity 3D

\subsection{Development phase}

The development phase consists of four modules - Image Modelling,Capture, Detection and Augment module.

\subsubsection{Image Modeling Module}

This phase consists of modeling the image in 3D form using Unity Software. The Design of the model will be stored in the database. 


\subsubsection{Capturing Module}

The input is image capture from a smartphone. This image will be analysed and recognized as an object. This module generates binary images i.e. a digital image that has two possible values for every pixel. Typically the 2 colours used for a binary image are black and white. The binary images are then processed to detect AR markers.

\subsubsection{Detection Module}

Detection of the marker will determine the position of the object. The tracking is done by calculating the relative position of the camera in real time. The vuforia detects and fetches 3D representations of particular images from the database.

\subsubsection{Augment Module}

The input for this module is calculated pose and $3 \mathrm{~d}$ object to augment. The renderer uses the calculated pose to combine the real image and the virtual component, and renders the enlarged image on the monitor of the mobile device.

\section{ALGORITHM USED - RANSAC}

The RANSAC algorithm is a training method that estimates model parameters by randomly sampling observation data.For data sets that contain both outliers and outliers, RANSAC uses a voting scheme to obtain the best results. The data elements in the data set are used to vote on one or more models. The RANSAC algorithm essentially consists of two steps, which are repeated iteratively.

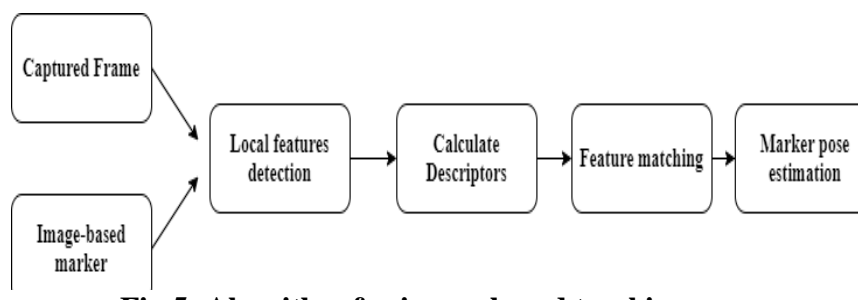

Fig 5: Algorithm for image-based tracking

The first step is to randomly select a sample subset with the fewest data elements from the input data set.The fitted model and the corresponding model parameters are calculated using only the elements of the sample. The size of the sample subset should be sufficient to determine the parameters of the model.

In the second step, the algorithm checks which elements of the entire data set match the model generated using the estimated parameters of the model obtained in the first step.

If a data item does not match the fitted model generated by a set of estimated model parameters within the error threshold that defines the maximum variance associated with noise, it is considered an outlier.

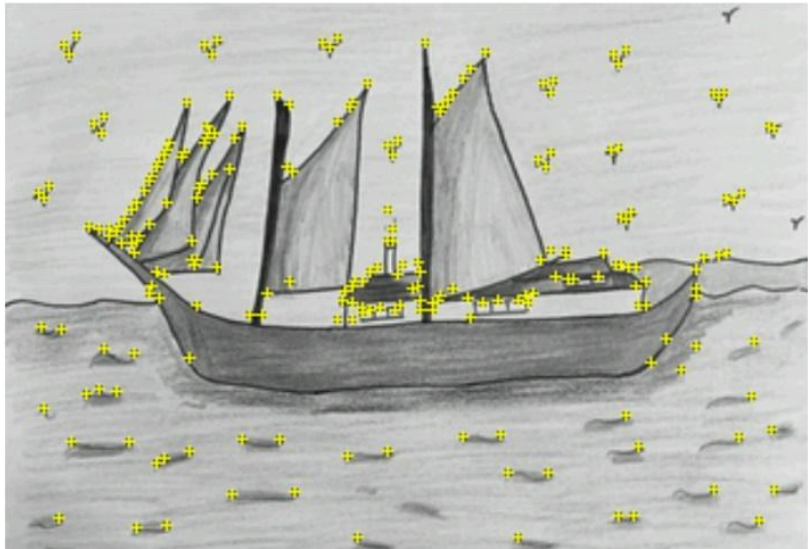

Fig 6: groups of inliers

\section{RESULTS AND DISCUSSIONS}

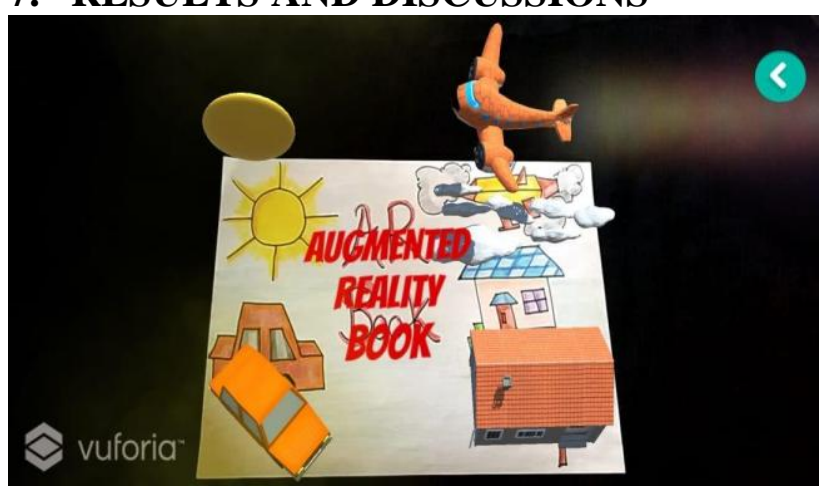

Fig. 7: ARBook Screen

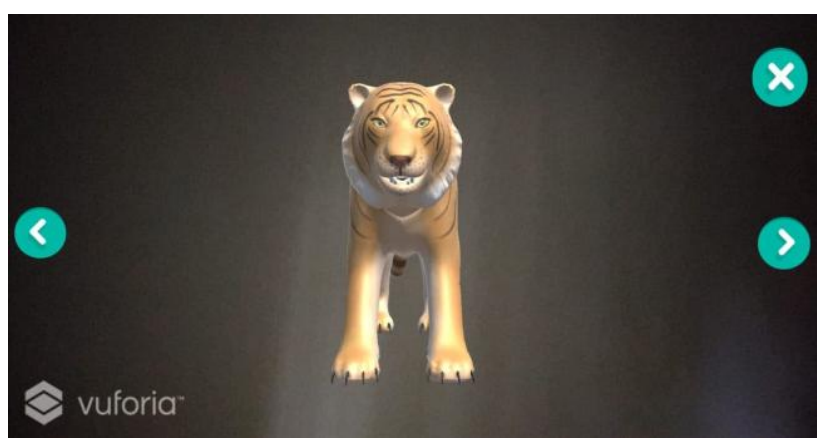

Fig 8: Augmentation of Animal

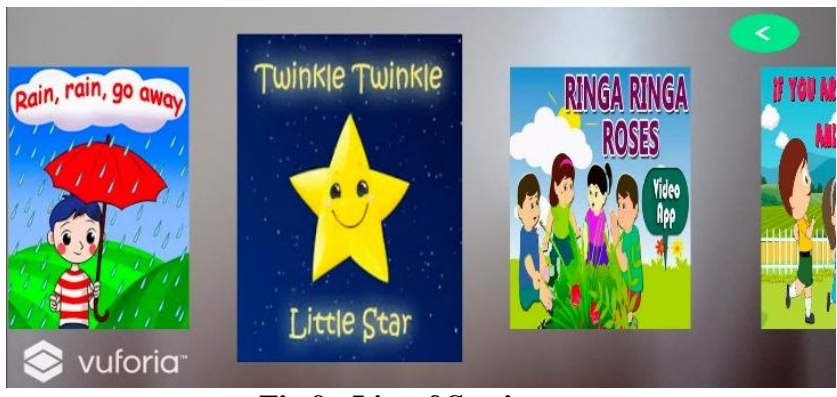

Fig 9: List of Stories 


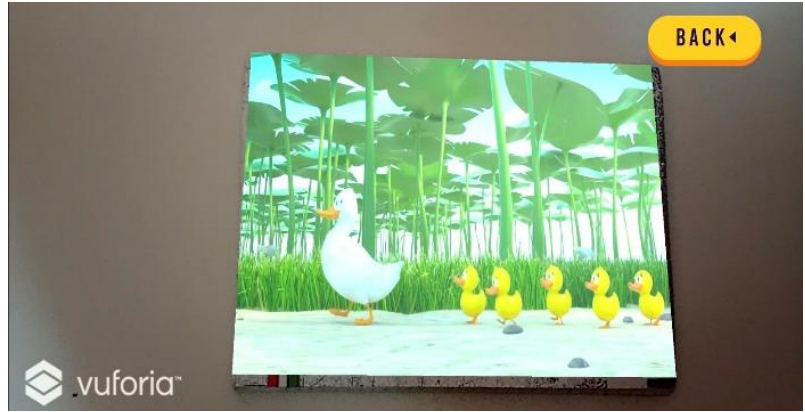

Fig 10: Augmentation of Stories

Fig.7. this is one of the ARBook images where User has to scan markers by smart device and the augmented form of that image will be shown to the user.

Fig.8.This is one of the animal pictures from the Animals category shown in augmented form with the background voice which will describe that animal and voice of a particular animal.

Fig.9. these are lists of stories where users can select any of the story videos and it will be played on a target marker.

Fig.10. this is an augmented form of story video selected by the user.

The Unity 3D application bundled with the Vuforia library is used for AR development.The application is easy to use. The user turns on the AR camera and automatically displays the object according to the default marker.Then the 3-dimensional object will appear on the smartphone. The mark recognition process is taken from a database created by the Vuforia library, which contains the mark image used to identify the image on the mark.

Marker cards are employed in the usage of the application. The smartphone was brought close to a marker that has been determined so that it could be read on the application. If the marker used isn't smart, then application can fail to observe and $3 \mathrm{~d}$ objects won't seem.

\section{CONCLUSION}

This paper proposes a combination of marker based and marker less augmented reality application using windows operating system which will help to combine virtual objects with the real environment facilitating various applications as mentioned in this paper. The main advantage is use of low cost devices such as smartphones as compared to the costly head mounted display devices. Secondly with the help of this project the students can clear theirsbasic, make visualization strong and experience the enjoyable environment.

\section{REFERENCES}

[1] Chen, Ruo,Chan, Kan Kan, "flashcards to learn vocabulary in early childhood education"-Journal of Educational Computing Research.2019

\section{$10.1177 / 07356331198$}

[2] Omar, Marlissa, et al-Effects of Mobile Augmented Reality (MAR) towards students visualization skills when learning orthographic projection,International Journal of Emerging Technologies in Learning (iJET) 14.20 (2019): 106-119.

[3] M. K. Mokhtar, F. Mohamed, M. S. Sunar, M. A. M. Arshad and M. K. MohdSidik-Development of MobileBased Augmented Reality Colouring for Preschool Learning,2018 e-Management and e-Services (IC3e), 2018, pp. 11-16, doi: 10.1109/IC3e.2018.8632639.

[4] Lu, Yuzhu, and Shana Smith, "Augmented reality ecommerce assistant system: trying while shopping",International Conference on Human-Computer Interaction. Springer, Berlin, Heidelberg, 2007.

[5] Bistaman, IzwanNurli Mat, Syed Zulkarnain Syed Idrus, and Salleh Abd Rashid, "The use of augmented reality technology for primary school education in Perlis, Malaysia"-In Journal of Physics: Conference Series, vol. 1019, no. 1, p. 012064. IOP Publishing, 2018.

[6] Jonathan Fernandes, Joseph Narasisa, Kevin Fernandes, SreenivasaB.1, "Marker based ar for a better understanding of visual learning"-Special Issue SACAIM - November 2016, 520-527.

[7] HelenPapagianisbookAugmentedHuman-How Technology Is Shaping the New Reality.

[8] John Tinnell and Sean Morey-Augmented Reality: "Innovative Perspectives across Art, Industry, and Academia".

[9] Ishaan BholaMagic-How Augmented Reality Will Change Our World".

[10] Silva, R., Oliveira, J.C. and Giraldi, G.A.-Introduction to augmented reality,National laboratory for scientific computation, 11 .

[11] Chang, G., Morreale, P. and Medicherla, P., 2010, March-Applications of augmented reality systems in education,In Society for Information Technology \& Teacher Education,International Conference

[12] Billinghurst, Mark, and Hirokazu Kato-Collaborative augmented reality,Communications of the ACM 45.7 (2002):64-70.

[13] Juan, C., Beatrice, F. and Cano, J., 2008, July-An ar system for learning the interior of the human body,Eighth IEEE International Conference on Advanced Learning Technologies (pp. 186-188).

[14] Yovcheva, Z, Buhalis, D. and Gatzidis, CSmartphone augmented reality applications for tourism.E-review of tourism research. 